\title{
Mitochondrial DNA Haplotypes Influence Energy Metabolism across Chicken Transmitochondrial Cybrids
}

\author{
Minghua Kong ${ }^{1}$, Hai Xiang ${ }^{2} \mathbb{D}$, Jikun Wang ${ }^{3}$, Jian Liu ${ }^{4}$, Xiben Zhang ${ }^{4,5}$ and Xingbo Zhao ${ }^{1,4, *(\mathbb{D})}$ \\ 1 National Engineering Laboratory for Animal Breeding; Key Laboratory of Animal Genetics, Breeding and \\ Reproduction, Ministry of Agriculture; College of Animal Science and Technology, China Agricultural \\ University, Beijing 100193, China; kmh928335059@163.com \\ 2 Guangdong Provincial Key Laboratory of Animal Molecular Design and Precise Breeding, School of Life \\ Science and Engineering, Foshan University, Foshan 528225, China; vamyluo@126.com \\ 3 Key Laboratory of Qinghai-Tibetan Plateau Animal Genetic Resource Reservation and Utilization, \\ Sichuan Province and Ministry of Education, Southwest Minzu University, Chengdu 610041, China; \\ xdzwjk@163.com \\ 4 Guizhou Nayong Professor Workstation, Bijie 553300, China; liu123985442@126.com (J.L.); \\ 13308579937@163.com (X.Z.) \\ 5 Institute of Animal Husbandry and Veterinary Medicine, Bijie 551700, China \\ * Correspondence: zhxb@cau.edu.cn; Tel.: +86-010-62733417; Fax: +86-010-62733417
}

Received: 2 December 2019; Accepted: 10 January 2020; Published: 16 January 2020

\begin{abstract}
The association between mitochondrial DNA haplotype and productive performances has been widely reported in chicken breeds. However, there has not been physiological evidence of this seen previously. In this study, chicken transmitochondrial cells were generated using the nucleus of the DF-1 cell line and mitochondria of primary cell lines derived from two native chicken breeds, Tibetan chicken and Shouguang chicken. Generally, Tibetan chicken primary cells showed a stronger metabolic capacity than Shouguang chicken primary cells. However, the Tibetan chicken cybrids had a dramatic drop in relative mtDNA copies and oxygen consumption. Higher rates of oxygen consumption (OCR) and expression levels of mitochondrial biogenesis and fusion genes were observed in Shouguang chicken cybrids, potentially reflecting that the mitochondrial DNA haplotype of Shouguang chicken had better coordination with the DF-1 nucleus than others. Meanwhile, mitonuclear incompatibility occurred in Tibetan chicken cybrids. The results demonstrate functional differences among mitochondrial DNA haplotypes and may shed light on the interaction between the mitochondria and nucleus in Gallus gallus domesticus.
\end{abstract}

Keywords: mitochondrial DNA haplotype; cybrids; energy metabolism; chicken breed

\section{Introduction}

The mitochondrion occupies a crucial position in almost all multicellular organisms, principally involved in the conversion of energy through respiratory chains [1]. In addition to cellular energy production, the mitochondrion houses essential pathways of heme synthesis, signal transduction, and apoptosis [2].

For millions of years, mitochondrial DNA (mtDNA) haplotypes have evolved, and certain mtDNA variants have been inherited [3]. Not only do mtDNA haplotypes vary among breeds and strains, but they confer positive and negative advantages to the organisms. For instance, mtDNA haplotypes influence the rate of radiographic OA progression and encephalomyopathies, adaptation to cold environments, and are responsible for the complex phenotypes of aging and longevity [3-6]. 
For animals, mtDNA haplotypes have been reported to associate with economically important traits, such as the milk quality of Holstein cows and reproductive capacity of pigs [7,8]. Many studies also indicate that mtDNA polymorphisms are correlated with growth traits, such as pectoral muscle fat content, duodenum length, Marek's disease resistance, and hypoxia adaptation in chicken breeds [9-11].

However, due to significant differences in the nucleus, environmental factors, and epigenetic modification, it has been challenging to evaluate the effect of the specific mtDNA haplotype on complex traits [12]. Moreover, the central point in defining the role of mitochondria in complex traits is to comprehensively understand the relationship between the nuclear genome and the mtDNA haplotype. At the organismal level, conplastic animals were generated by backcrossing females (mitochondrial donors) with males (nuclear donors) for over 20 generations; then, complex traits were studied, such as longevity in conplastic mice and fitness in conplastic Drosophila $[13,14]$. In order to study the genetic and metabolic consequences caused by different mtDNA haplotypes at the cellular level, cytoplasmic hybrids (cybrids) are created, where the mitochondria donor cells are enucleated and then transfer to the nuclear donor cells previously depleted of their own mtDNA. The cybrids generated through this strategy maintain the constant nuclear components, so the cellular changes caused by the transplanted mtDNA can be studied $[15,16]$.

China is a native country of chicken production, with a long cultivation history and abundant resources. Compared with commercial breeds, native chickens have many desirable characteristics, such as suitability to environments (hypoxic adaptation), disease resistance, and excellent meat quality. The Tibetan chicken is a native breed that has inhabited the Qinghai-Tibet Plateau ( $4000 \mathrm{~m}$ above sea level on average) for thousands of years and has adapted well to the harsh environments, high altitude, hypoxia, and cold conditions [17]. Tibetan chickens are widely used in agricultural practices, providing local people with meat and eggs. However, egg and meat production of Tibetan chicken is much lower than the breed which lives in the plain, such as the Shouguang chicken, which has a significant advantage in body growth and egg production. Animal products, such as meat and eggs, can be considered as a transformation from chemical energy through mitochondrial metabolism [18].

In this study, we chose the DF-1 cell line [19] as the nuclear donor, and primary embryo fibroblast cell lines from Tibetan and Shouguang chicken as mitochondria donors, to generate cybrid cells. These cybrids harboring different mtDNA haplotypes displayed variable expressions of genes associated with mitochondrial biogenesis and mitochondrial fusion. Most importantly, they presented significant differences in energy metabolism. Our study is the first to identify the effect of mtDNA haplotype on energy metabolism in Chinese native chicken breeds by using transmitochondrial cells.

\section{Materials and Methods}

\subsection{Cells Preparation and Culture Conditions}

All the experimental procedures followed the guidelines for animal management of China Agricultural University (CAU), and the experimental protocols were allowed by the Experimental Animal Care and Use Committee of CAU (approval code: CAU20151205-5).

The chicken embryo fibroblast cells (DF-1) from the East Lansing Line were gifted by Professor Zhao Yaofeng, China Agricultural University. Primary chicken embryo fibroblasts were isolated from two native chicken breeds, $T$ from Tibetan chicken and $S$ from Shouguang chicken. The growth medium used was DMEM supplemented with $10 \% \mathrm{FBS}, 100 \mu \mathrm{g} / \mathrm{mL}$ streptomycin, and $100 \mathrm{U} / \mathrm{mL}$ penicillin. For $\rho^{0}$ cells, an extra $50 \mu \mathrm{g} / \mathrm{mL}$ uridine was added into the medium. All the cells were cultured in $5 \%$ $\mathrm{CO}_{2} / 95 \%$ air at $37^{\circ} \mathrm{C}$.

\subsection{Generation of Cybrids}

Using Lipofectamine 2000 (Invitrogen, Carlsbad, CA, USA), plasmid p-eGFP-neo was transfected into DF-1 cells (D), which was used as a selection marker for successful cybrids. $\rho^{0}$ cells and enucleated mitochondrial donor cells were created according to the modified procedures of Bacman and Moraes [20]. 
DF-1 cells, the nuclear donor cell line $\left(\rho^{0}\right)$, were cultured with $2.5 \mu \mathrm{g} / \mathrm{mL}$ rhodamine $6 \mathrm{G}$ (R-6G) for five days. The other primary embryo cells ( $\mathrm{T}$ and $\mathrm{S}$ ) were used as mitochondria donor cells. Cybrids were created by the fusion of the $\rho^{0}$ cells with the enucleated cells using polyethylene glycol (PEG) $[20,21]$.

\subsection{Mitogenome Sequencing}

A total of 22 pairs of PCR primers were used to amplify the whole chicken mtDNA (Supplementary Table S1). Sequences were analyzed and submitted to GenBank. The polymorphisms were compared using MEGA6. The conservation of the missense mutations was evaluated by the Grantham Score [22-24].

\subsection{Metabolic Assays}

The specific medium for the mitochondrial stress test and glycolysis test was made, respectively. For the mitochondrial stress test, unbuffered DMEM supplemented with $2 \mathrm{mM}$ sodium pyruvate, $10 \mathrm{mM}$ D-glucose, and $4 \mathrm{mM}$ L-glutamine was used, and the $\mathrm{pH}$ was adjusted to 7.4. For the glycolysis test, unbuffered glucose-free DMEM containing $4 \mathrm{mM}$ L-glutamine was used, and the $\mathrm{pH}$ was adjusted to 7.35 .

The cells were cultured in 6 wells of XF96 cell culture microplates (Seahorse Bioscience) at the density of $1.8 \times 10^{4}$ cells per well. The sensor cartridge was hydrated by adding $200 \mu \mathrm{L}$ of XF calibrant solution to each well, and incubated in a $37^{\circ} \mathrm{C}, \mathrm{CO}_{2}$-free incubator overnight. The next day, the normal cell medium was replaced with the assay medium. For the mitochondrial stress test, reagents were injected by following the protocol of the Seahorse XF Cell Mito Stress Test kit. First, the "resting" OCR was established, and then oligomycin $(2.0 \mu \mathrm{M})$ was added. Oligomycin inhibits complex V (ATP synthesis), so the difference before and after oligomycin can be calculated as the OCR linked to ATP production. Subsequent injection of FCCP $(0.25 \mu \mathrm{M})$, which is an uncoupler, was used to indicate the maximum rate of respiration that the cell can achieve. Finally, rotenone $(1 \mu \mathrm{M})$ and antimycin $(1 \mu \mathrm{M})$ were added to inhibit complex I and complex III to shut down the respiration chain. Thus, non-mitochondrial respiration can be calculated. For glycolysis analyses, reagents were injected by following the protocol of the Seahorse Glycolysis Stress Test kit. First, glucose (10 mM) was added. Then, oligomycin $(2 \mu \mathrm{M})$ was injected to establish the "highest" ECAR by shifting the energy generation to glycolysis. The last injection is 2-DG $(100 \mathrm{mM}))$ measured the non-glycolytic ECAR. The results were normalized by the CyQUANT Cell proliferation kit (Invitrogen). OCR was shown as pmol $\mathrm{O}_{2} / \mathrm{min} / 1.8 \times 10^{4}$ cells; ECAR was shown as $\mathrm{mpH} / \mathrm{min} / 1.8 \times 10^{4}$ cells.

\subsection{Total DNA Isolation and Relative mtDNA Copies Detecting}

High-quality genomic DNA was extracted from all the cell lines and the relative mtDNA copies were determined using real-time PCR [25]. A pair of primers (Forward 5'-3': CCAACCACCAACCTGATAGC; Reverse 5'-3': TGTGGGATGGAAGAGTGCC) was designed to target the ND4 of mtDNA (KM433666.1). The 18S rRNA gene was used for standardization (Forward 5'-3': ATAACGAACGAGACTCTGGCA 3'; Reverse 5'-3': CGGACATCTAAGGGCATCACA). The optimum thermal cycling parameters were $95^{\circ} \mathrm{C}$ for $15 \mathrm{~min}, 40$ cycles of $95{ }^{\circ} \mathrm{C}$ for $10 \mathrm{~s}, 58^{\circ} \mathrm{C}$ for $20 \mathrm{~s}$, and $72{ }^{\circ} \mathrm{C}$ for $25 \mathrm{~s}$. The lengths of the amplification product were $157 \mathrm{bp}$ and $136 \mathrm{bp}$, respectively. The cycle threshold $\left(C_{T}\right)$ was used for calculations. The $\Delta C_{T}$ was calculated by the subtraction of the average $18 \mathrm{~S}$ rRNA $\mathrm{C}_{\mathrm{T}}$ value from the average $\mathrm{ND} 4 \mathrm{C}_{\mathrm{T}}$ value. We used the cybrid $\mathrm{D}+\mathrm{D}$ to normalize and subtracted the $\Delta C_{T}$ of $D+D$ from the $\Delta C_{T}$ of each group, yielding the value of $\Delta \Delta C_{T}$. The fold change of relative mtDNA copies were detected with the $2^{-\Delta \Delta C T}$ method [26].

\subsection{Total RNA Extraction and $q P C R$}

Total RNA was extracted from all the cell lines by using the RNeasy Mini Extraction (Qiagen) and RNase-Free DNase Set (Qiagen). Then $500 \mathrm{ng}$ of RNA was taken to do reverse transcription by using the QuantiTect Reverse Transcription Kit (Qiagen) for Q-PCR analyses. The SYBR Green PCR Master 
Mix (Tiangen) and Bio-Rad iCycler iQ5 detection system were used to perform the Q-PCRs. The cybrid $\mathrm{D}+\mathrm{D}$ was used as the standard, and the expression levels were calculated with reference to expression of $\beta$-actin. The results were shown as a fold change using the $2^{-\Delta \Delta C T}$ method [26]. The sequences of all the primers were listed in Supplementary Table S2.

\subsection{Statistical Analysis}

Statistical analyses were performed using SPSS 20.0 software (SPSS Inc., Chicago, IL, USA). Tukey's multiple comparison test of ANOVA was used to detect the main effect of the mtDNA haplotypes. Data are exhibited as means \pm SEM.

\section{Results}

\subsection{Generation of Cybrids}

DF-1 fibroblast cells (D) were cultured in medium containing rhodamine 6G (R-6G) to create $\rho^{0}$ cells. Primary embryo fibroblast cells from Tibetan chicken (T) and Shouguang chicken (S) were used as mitochondrial donor cells. We found that endogenous mtDNA was substituted for exogenous mtDNA completely by PCR-amplifying the mtDNA control regions after clone selection. Results showed that cybrids (D+T, D+S) only contained the Tibetan chicken (T) and Shouguang chicken (S) mtDNA, respectively. The endogenous mtDNA (D) cannot be detected after five weeks (Figure 1).
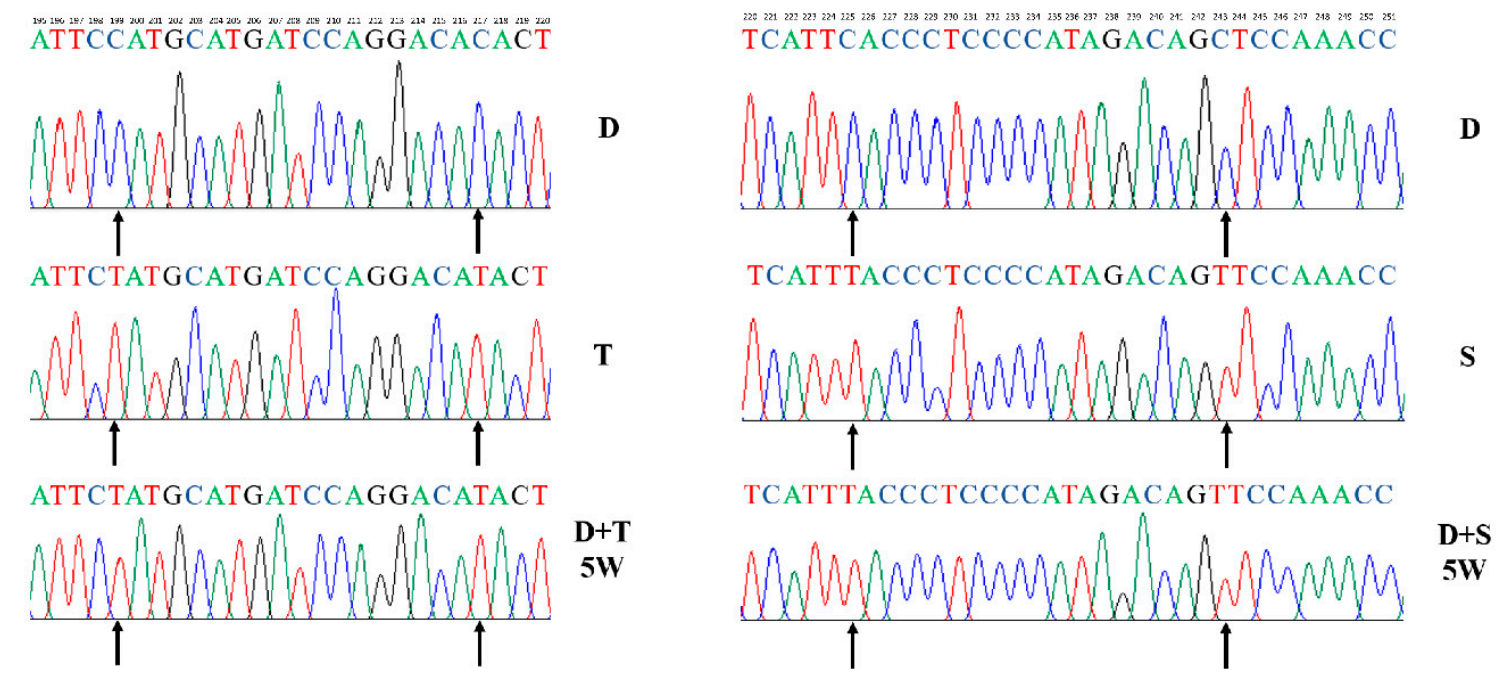

Figure 1. Sequence verification in the generation of different cybrid cells. D, T, and S harbor characteristic sequence signatures within the D-loop region. The endogenous mtDNAs (D) were no longer detectable in the fifth week $(\mathrm{D}+\mathrm{T} / 5 \mathrm{~W}, \mathrm{D}+\mathrm{S} / 5 \mathrm{~W})$. In cybrid cells, the acronym $\mathrm{D}$ denotes a common nucleus of $\mathrm{DF}-1$, and $+\mathrm{T}$ and $+\mathrm{S}$ represent the source of the mitochondria. $5 \mathrm{~W}$ : cell culture for five weeks.

\subsection{Mitogenome Sequencing}

The mtDNA haplotypes of all the chicken cell lines (D, T, and S) were sequenced and submitted to GenBank with accession numbers (MK163561-MK163563). After determining the mtDNA sequence of the cybrids $(D+D, D+T$, and $D+S)$, we found that the mtDNA sequences of each cybrid cell were identical to the mitochondrial donor cells (Figure 1).

The polymorphisms of the three mtDNA haplotypes were summarized in Table 1. Specifically, the alignment T/S harbored 41 variations, while D/T and D/S presented 39 and 46 variations, respectively. T/S showed 25 mutations in the 13 mtDNA peptide-coding genes, including five missense mutations, of which one had conservative Grantham scores and four had moderately conservative Grantham scores [22,23]. D/T and D/S presented five and four missense mutations, respectively. More details of the polymorphisms were listed in Supplementary Table S3 and S4. 
Table 1. Mitogenome divergences among the mitochondrial haplotypes.

\begin{tabular}{cccccccc}
\hline \multirow{2}{*}{ Comparison } & \multicolumn{4}{c}{ SNPs/InDels } & AA & Grantham Score \\
\cline { 2 - 5 } & D-loop & rRNA & tRNA & Protein & Total & Change & Summary \\
\hline D/T & 9 & 2 & 1 & 26 & 39 & 5 & 1 in 0-50, 4 in 51-100 \\
D/S & 10 & 4 & 2 & 29 & 46 & 4 & 2 in 0-50, 2 in 51-100 \\
T/S & 11 & 4 & 1 & 25 & 41 & 5 & 1 in 0-50, 4 in 51-100 \\
\hline
\end{tabular}

\subsection{Metabolic Assays}

The Seahorse XF96 Analyzer was used to quantify oxidative phosphorylation (OXPHOS) and glycolysis of chicken primary cells and cybrid cells. Following the protocol with specific components, the level of OXPHOS and glycolysis was evaluated by the mitochondrial stress test and glycolysis stress test, respectively.

For primary cells, T generally showed higher basal respiration, ATP production $(p<0.05)$, and maximal respiration than $\mathrm{S}$ (Figure 2A). T also showed higher ECAR than $\mathrm{S}$, with higher glycolysis and glycolytic reserve $(p<0.01)$ (Figure 3A). It indicated that Tibetan chicken cells have a higher rate of anaerobic respiration due to their environment. In contrast, the Tibetan chicken cybrid (D+T) showed lower OCRs than the Shouguang chicken cybrid, with lower basal respiration $(p<0.01)$, ATP production $(p<0.01)$, and maximal respiration $(p<0.01)$ (Figure $2 \mathrm{~B})$. These results indicated the inferior capacity in mitochondrial function and energy metabolism in the Tibetan chicken cybrid. The Shouguang chicken cybrid $(D+S)$ also presented a higher OCR than $D+D$, with higher basal respiration $(p<0.05)$, ATP production $(p<0.01)$, and maximal respiration $(p<0.01)$ (Figure $2 \mathrm{~B})$, demonstrating a significant increase in energy metabolism. The results above can allow us to measure the metabolic properties of the chicken primary cells and cybrid cells.
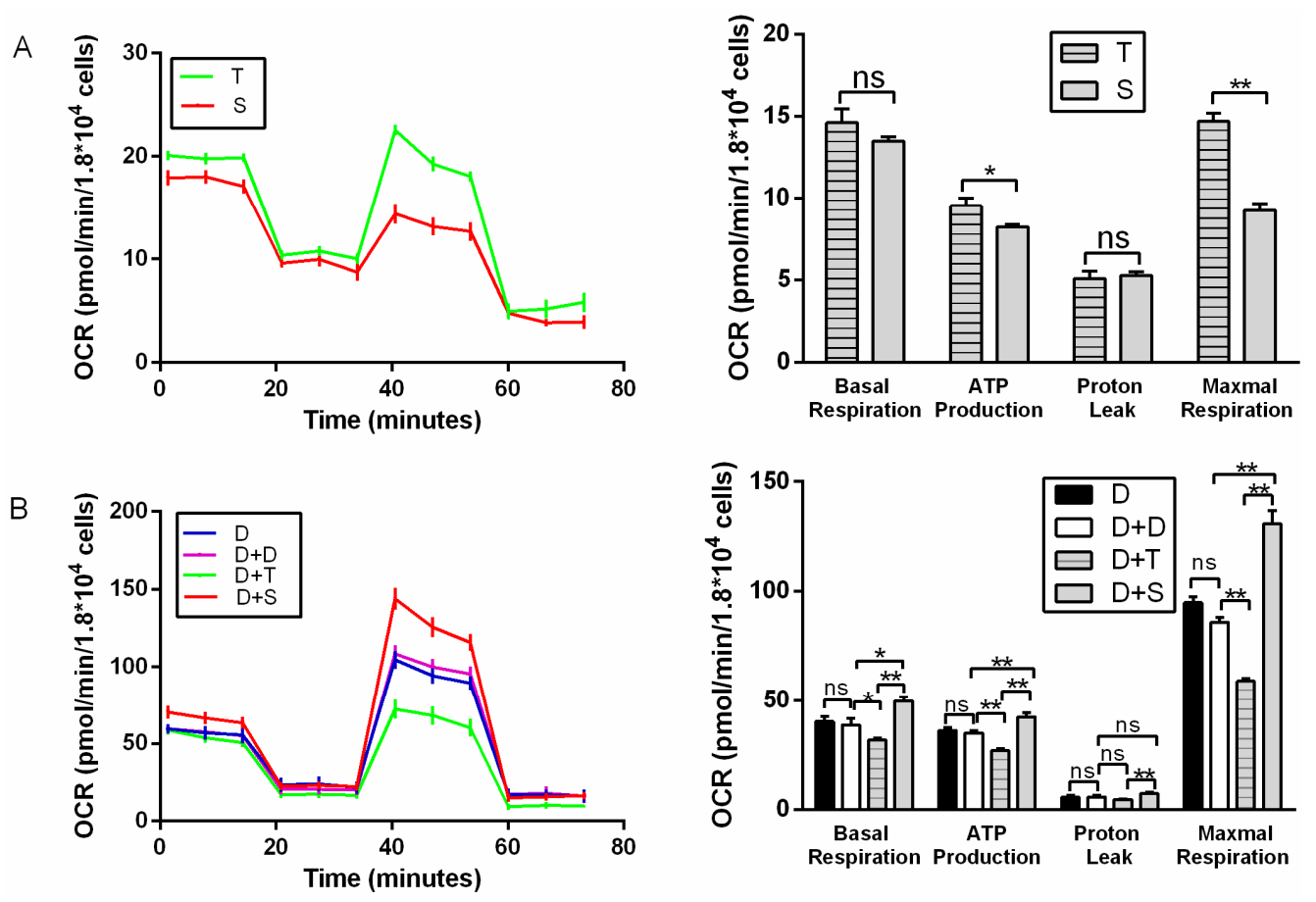

Figure 2. Mitochondrial stress test for primary chicken cells and cybrids. (A) Illustrating OCR of primary cell lines (T, S). (B) Illustrating OCR of cybrid cells; D was used as a control for D+D. Oligomycin, FCCP, and rotenone plus antimycin A were injected sequentially to the cells. OCR profiles were expressed as pmole $\mathrm{O} 2 / \mathrm{min} / 1.8 \times 10^{4}$ cells. In cybrid cells, the acronym D denotes a common nucleus of DF-1 cell, and $+\mathrm{T}$ and $+\mathrm{S}$ represent the source of mitochondria. $n=6$ per group. ${ }^{*}$ means $p<0.05,{ }^{* *}$ means $p<0.01$, and ns means $p>0.05$. 
A

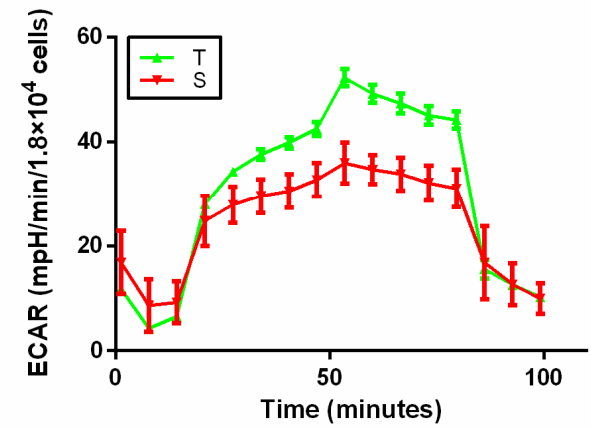

B

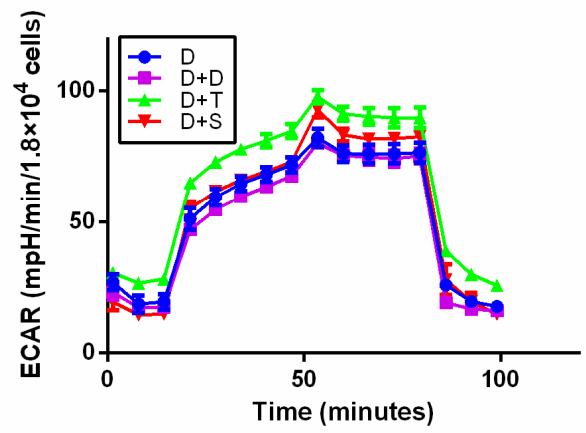

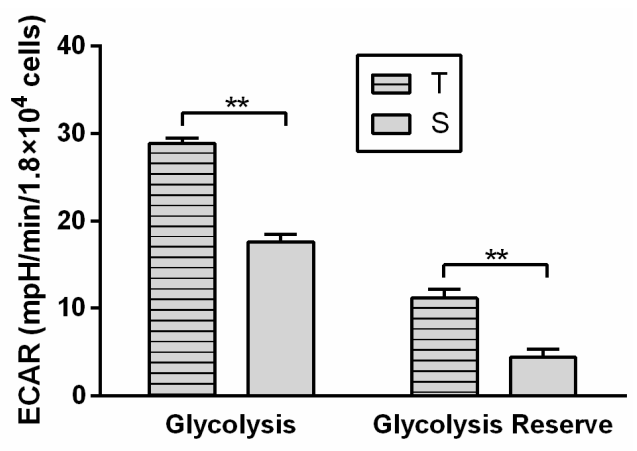

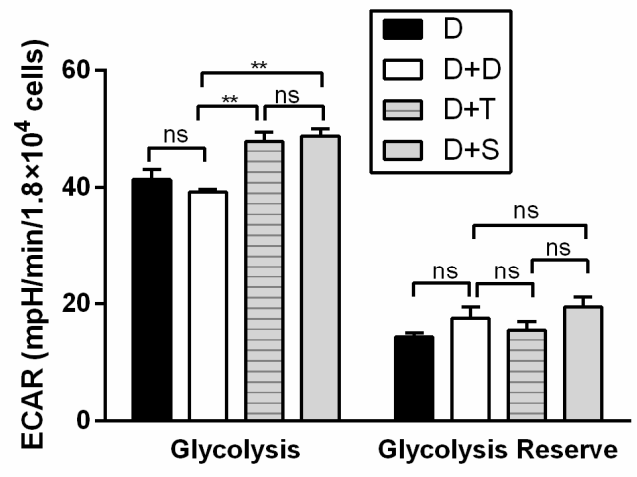

Figure 3. Glycolysis test for primary chicken cells and cybrids. (A) Illustrating ECAR of primary cell lines (T and S). (B) Illustrating ECAR of cybrids, D was used as a control for D+D. All cells exposed sequentially to glucose, oligomycin, and 2-DG. ECAR profiles were expressed as $\mathrm{mpH} / \mathrm{min} / 1.8 \times 10^{4}$ cells. $n=6$ per group. ${ }^{* *}$ means $p<0.01$ and ns means $p>0.05$.

\subsection{Relative mtDNA Copies Detecting}

mtDNA copy number of primary chicken cells and cybrids were detected and calculated as $2^{-\triangle \Delta C T}$ according to the qPCR analysis (Figure 4). In the study, the primary Tibetan chicken embryo cells (T) carried higher mtDNA copies than the Shouguang chicken primary cells $(S)(p<0.01)$. However, the Tibetan chicken cybrid (D+T) harbored the lowest amounts of mtDNA among all cybrids $(p<0.01)$. However, Shouguang cybrid $(\mathrm{D}+\mathrm{S})$ had a significantly higher amount of mtDNA than $\mathrm{D}+\mathrm{D}(p<0.01)$.

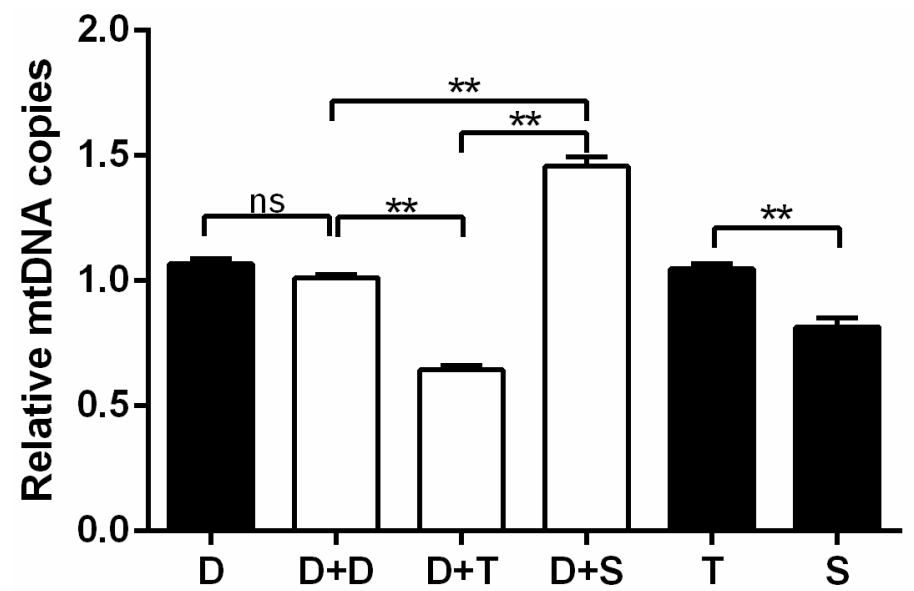

Figure 4. Measurement of relative mtDNA copies in primary cells and cybrids. Black column, DF-1 cells and primary cells; white columns denote cybrids. $n=3$ per group. ${ }^{* *}$ means $p<0.01$ and ns means $p>0.05$. 


\subsection{Expression Levels of Genes Involved in Mito-Biogenesis and Mito-Fusion}

The expression of four genes (NRF1, NRF2, TFAM, PGC1- $\alpha$ ) was used to measure mitochondrial biogenesis (Figure 5). D and D+D showed the consistent tendency of expression in the four genes. D+T exhibited lower expression levels in NRF1 and PGC1- $\alpha$ than the other cybrids $(p<0.01)$. No significant difference was observed between $\mathrm{D}+\mathrm{D}$ and $\mathrm{D}+\mathrm{T}$ in TFAM. D+S illustrated a higher level of expression than D+D in PGC1- $\alpha$ and TFAM expression levels ( $p<0.05$ and $p<0.01$, respectively). There was no significant change between $\mathrm{D}+\mathrm{T}$ and $\mathrm{D}+\mathrm{S}$ in NRF2. For primary embryonic cells, $\mathrm{T}$ presented higher expression levels of NRF1 and TFAM than $S$ ( $p<0.05, p<0.01$, respectively), similar expression levels of the other two genes observed.
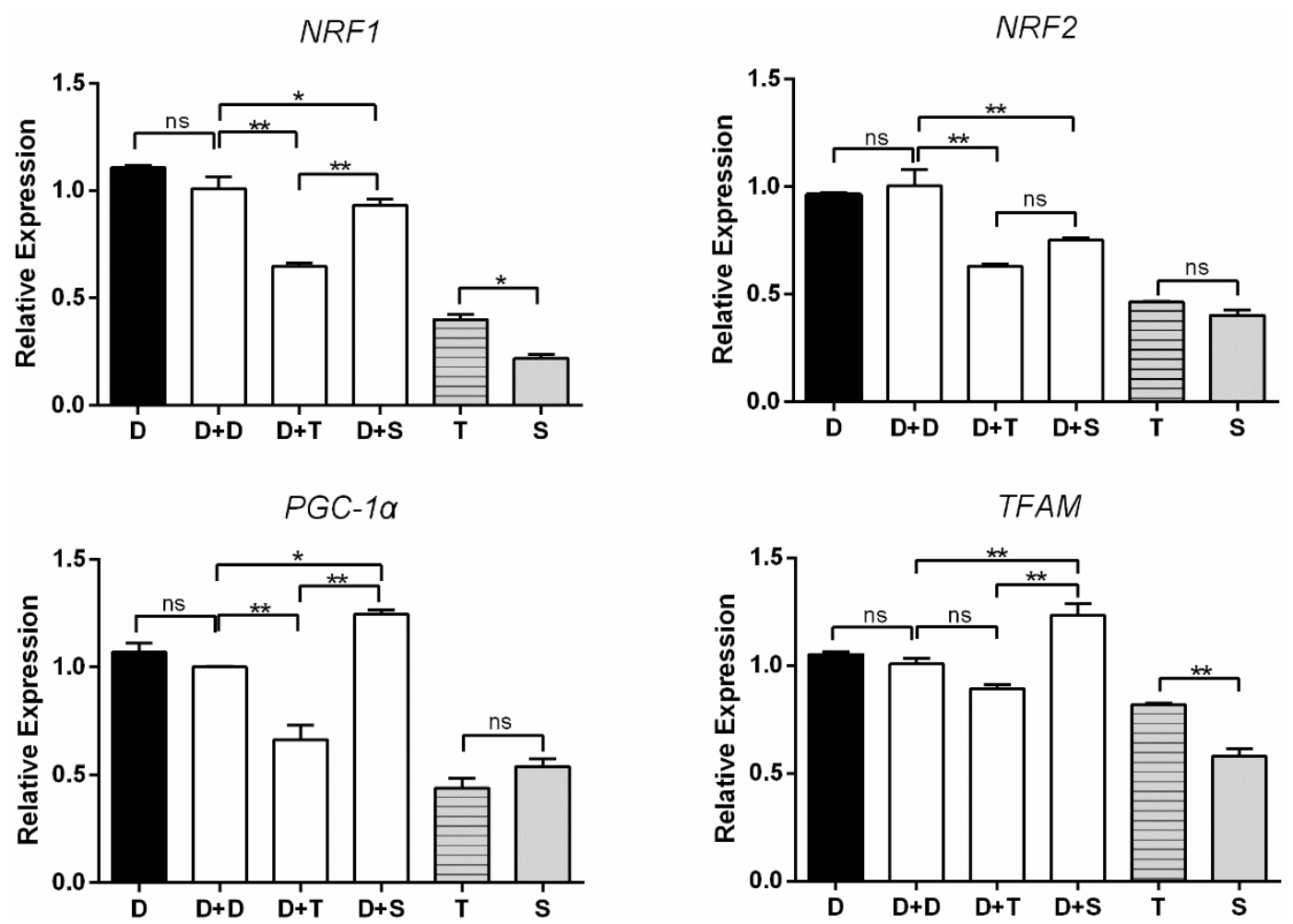

Figure 5. Expression levels of four mitochondrial biogenesis-related genes in primary cells and cybrids. Black column: DF-1 cells and primary cells; white columns denote cybrids. $n=3$ per group. * means $p<0.05,{ }^{* *}$ means $p<0.01$, and ns means $p>0.05$.

The expression levels of OPA1, MFN1, and MFN2 were used to measure the mitochondrial fusion (Figure 6). Similar to the evaluated genes above, $\mathrm{D}$ and $\mathrm{D}+\mathrm{D}$ have constant expression levels in all analyses. For cybrids, D+T showed lower expression levels of OPA1 and MFN1 than D+D $(p<0.01$ and $p<0.05$, respectively), and no significant change was observed between $\mathrm{D}+\mathrm{T}$ and $\mathrm{D}+\mathrm{D}$ in MFN2. For MFN1 and MFN2, D+S had higher expression levels than D+D ( $p<0.05$ and $p<0.01$, respectively), and a similar expression level was observed between D+S and D+D for OPA1. For primary cells, $\mathrm{T}$ presented similar expression levels to $\mathrm{S}$ for these three genes. 

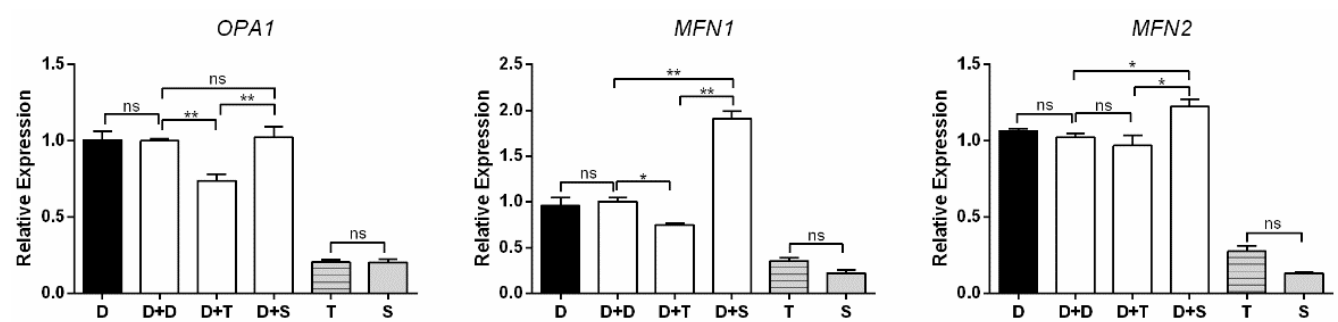

Figure 6. Expression levels of three mitochondrial fusion-related genes in primary cells and cybrids. Black columns: DF-1 cells and primary cells; white columns denote cybrids. $n=3$ per group. ${ }^{*}$ means $p<0.05,{ }^{* *}$ means $p<0.01$, and ns means $p>0.05$.

\section{Discussion}

Various mtDNA haplotypes have developed during millions of years of evolution. Previous studies have shown that the polymorphisms in mtDNA were associated with many different phenotypes, such as human disease and animal productive traits $[6,8,10]$. By generating cybrids, in which the mitochondria from one cell line are transferred into a $\rho^{0}$ cell line, direct evidence can be shown to explain the association. For example, having the human mtDNA haplotype $\mathrm{J}$ is beneficial in decreasing the rate of incident knee osteoarthritis over time [27], while haplotype $\mathrm{H}$ decreases the risk for age-related macular degeneration [28]. Furthermore, the mechanism of interaction between the mitochondria and nucleus can be studied.

Compared to Shouguang chicken (S), Tibetan chicken (T) showed a higher amount of mtDNA in primary cells. Moreover, Tibetan chicken primary cells presented higher basal respiration, ATP production, and maximal respiration (Figure 2A), which allows us to infer a biological adaptation to the harsh environment and resistance to the cold condition. Tibetan chicken cells exhibited higher glycolysis and glycolysis capacity (Figure 3A). A previous study had already suggested that the Tibetan chicken has higher glucose oxidation, mitochondrial metabolism, and antioxidant ability [29]. Combined with our results, the huge differences seen between the Tibetan chicken and Shouguang chicken indicate an adaptation to the hypoxic environment of the Tibetan chicken.

Compared to other chicken cybrids $(\mathrm{D}+\mathrm{D}, \mathrm{D}+\mathrm{S})$, the cybrid from the Tibetan chicken $(\mathrm{D}+\mathrm{T})$ manifested generally lower OCR indexes (basal respiration, ATP production, and maximal respiration), mtDNA copy number, and mRNA expression of genes involved in mito-biogenesis and mito-fusion, but showed a higher ECAR index (glycolysis and glycolysis reserve). Tibetan chicken primary cells (T) exhibited a higher rate of ATP production than others, since higher mtDNA copies were detected in Tibetan chicken primary cells (Figure 4). However, the metabolic level per mitochondrion in Tibetan chicken primary cells was similar to the mitochondrion of Shouguang chicken primary cells based on the mitochondrial content differences. However, Tibetan chicken cybrid appeared "weaker" in mitochondrial metabolism. So, to keep normal physical activities, the cybrid produced the ATP through glycolysis as a compensatory mechanism, and that may be the reason the Tibetan cybrid (D+T) harbored a similar ECAR to the Shouguang cybrid (D+S) (Figure 3B). Due to most proteins in the five complexes of the electron transfer chain being encoded by nuclear DNA and then transferred into the mitochondrion, it is required that mitochondria maintain coordinated communication with the nucleus [30]. The DF-1 cell line was derived from the East Lansing Line, a lowland breed. And the alignment D/S only had two missense values that are moderately conserved, whereas the alignment $\mathrm{D} / \mathrm{T}$ had four missense values that are moderately conserved (Table 1). It indicated that the combination of the D nucleus and $T$ mtDNA haplotype was more divergent than the Shouguang cybrids $(D+S)$. These results support the hypothesis of mitochondria-nuclear incompatibility and mitochondria-nuclear coevolution [31,32].

NRF1, NRF2, TFAM, and PGC-1 $\alpha$ are involved in the critical pathway of mitochondrial biogenesis and metabolic processes [33,34]. PGC-1 $\alpha$ is an essential transcriptional coactivator in inducing mitochondrial biogenesis by activating nuclear respiratory factor 1 (NRF1), nuclear respiratory factor 2 (NRF2), and other transcription factors. NRF1 and NRF2 can activate mitochondrial transcription 
factor A (TFAM) to modulate the mitochondrial activity [33]. OPA1, MFN, and MFN2 are mito-fusion genes. Specifically, OPA1 mediate the fusion of the inner membrane, and MFN1 and MFN2 modulate the fusion of the outer membrane. The fusion of mitochondria induces supercomplexes of the ETC, maximizing OXPHOS activities $[35,36]$. In this study, among these cybrids, the Shouguang cybrid $(\mathrm{D}+\mathrm{S})$ harbored higher expression levels of these genes (Figures 5 and 6). Meanwhile, D+S exhibited a generally higher OCR than other cybrids, with higher basal respiration, ATP production, and reserve capacity (Figure 2B), reflecting an active energy metabolism. Since all these genes are encoded by the nuclear genome, the results suggest that the expression of these genes could be regulated by the mtDNA haplotype. Previous studies also showed that with the same nuclear genome, the mtDNA haplotype can regulate the transcription of the nuclear genes by methylation [37]. These results suggested that haplotype $\mathrm{S}$ could communicate more effectively with the nucleus of the DF-1 cell than haplotype T and D. Moreover, a broad range of interactions between the genomes does not only depend on the subunit of protein complexes but also rely on the protein/RNA and protein/DNA interplay [38].

\section{Conclusions}

In conclusion, our study provides the first evidence that under the same nuclear background, specific mtDNA haplotypes can induce functional differences in cellular energy metabolism in Gallus gallus domesticus. Our findings explain the associations between the mtDNA variants and specific traits, and may give insight into the effects of mtDNA haplotypes on the metabolic phenotypes of farm animals.

Supplementary Materials: The following are available online at http://www.mdpi.com/2073-4425/11/1/100/s1, Table S1: Primer pairs for the amplification of chicken mitochondrial DNA sequences, Table S2: Primer pairs for real-time PCR, Table S3: Detailed variant sites among three chicken mitogenomes, Table S4: Detailed information of missense mutations.

Author Contributions: Conceptualization, X.Z. (Xingbo Zhao) and H.X.; investigation, M.K. and J.W.; formal analysis, M.K., H.X., J.L. and X.Z. (Xiben Zhang); writing-original draft preparation, M.K.; writing一review and editing, X.Z. (Xingbo Zhao); funding acquisition: X.Z. (Xingbo Zhao). All authors have read and agreed to the published version of the manuscript.

Funding: This research was funded by the Joint Projects of Guizhou Nayong Professor Workstation, "201705510410352" and the Yunnan Special Fund for Science and Technology Development, "2018IB016".

Acknowledgments: We thank Yaofeng Zhao, China Agricultural University, for the donation of the DF-1 cell line; Miss Inna Sirota, Cornell University, for proofreading assistance.

Conflicts of Interest: The authors declare no conflict of interest.

\section{References}

1. Chinnery, P.F.; Hudson, G. Mitochondrial genetics. Brit. Med. Bull. 2013, 106, 135-159. [CrossRef] [PubMed]

2. Van der Giezen, M.; Tovar, J. Degenerate mitochondria. EMBO Rep. 2005, 6, 525-530. [CrossRef]

3. Ruiz-Pesini, E.; Mishmar, D.; Brandon, M.; Procaccio, V.; Wallace, D.C. Effects of purifying and adaptive selection on regional variation in human mtDNA. Science 2004, 303, 223-226. [CrossRef] [PubMed]

4. Goto, Y.; Nonaka, I.; Horai, S. A mutation in the tRNA (Leu) (UUR) gene associated with the MELAS subgroup of mitochondrial encephalomyopathies. Nature 1990, 348, 651-653. [CrossRef] [PubMed]

5. Bratic, A.; Larsson, N.G. The role of mitochondria in aging. J. Clin. Invest. 2013, 123, 951-957. [CrossRef]

6. Fernandez-Moreno, M.; Soto-Hermida, A.; Vazquez-Mosquera, M.E.; Cortes-Pereira, E.; Pertega, S.; Relano, S.; Oreiro-Villar, N.; Fernandez-Lopez, C.; Blanco, F.J.; Rego-Perez, I. A replication study and meta-analysis of mitochondrial DNA variants in the radiographic progression of knee osteoarthritis. Rheumatology 2017, 56, 263-270. [CrossRef]

7. Schutz, M.M.; Freeman, A.E.; Lindberg, G.L.; Koehler, C.M.; Beitz, D.C. The Effect of Mitochondrial-DNA on Milk-Production and Health of Dairy-Cattle. Livest. Prod. Sci. 1994, 37, 283-295. [CrossRef]

8. Tsai, T.S.; Rajasekar, S.; St. John, J.C. The relationship between mitochondrial DNA haplotype and the reproductive capacity of domestic pigs (Sus scrofa domesticus). BMC Genet. 2016, 17, 67. [CrossRef] 
9. Lu, W.W.; Hou, L.L.; Zhang, W.W.; Zhang, P.F.; Chen, W.; Kang, X.T.; Huang, Y.Q. Study on heteroplasmic variation and the effect of chicken mitochondrial ND2. Mitochondrial DNA Part A 2016, 27, 2303-2309.

10. Li, S.; Aggrey, S.E.; Zadworny, D.; Fairfull, W.; Kuhnlein, U. Evidence for a genetic variation in the mitochondrial genome affecting traits in White Leghorn chickens. J. Hered. 1998, 89, 222-226. [CrossRef]

11. Wang, X.Y.; He, Y.; Li, J.Y.; Bao, H.G.; Wu, C. Association of a missense nucleotide polymorphism in the MT-ND2 gene with mitochondrial reactive oxygen species production in the Tibet chicken embryo incubated in normoxia or simulated hypoxia. Anim. Genet. 2013, 44, 472-475. [CrossRef] [PubMed]

12. Paliwal, S.; Fiumera, A.C.; Fiumera, H.L. Mitochondrial-Nuclear Epistasis Contributes to Phenotypic Variation and Coadaptation in Natural Isolates of Saccharomyces cerevisiae. Genetics 2014, 198, 1251-1265. [CrossRef] [PubMed]

13. James, A.C.; Ballard, J.W. Mitochondrial genotype affects fitness in Drosophila simulans. Genetics 2003, 164, 187-194. [PubMed]

14. Latorre-Pellicer, A.; Moreno-Loshuertos, R.; Lechuga-Vieco, A.V.; Sanchez-Cabo, F.; Torroja, C.; Acin-Perez, R.; Calvo, E.; Aix, E.; Gonzalez-Guerra, A.; Logan, A.; et al. Mitochondrial and nuclear DNA matching shapes metabolism and healthy ageing. Nature 2016, 535, 561-565. [CrossRef] [PubMed]

15. Trounce, I.; Wallace, D.C. Production of transmitochondrial mouse cell lines by cybrid rescue of rhodamine-6G pre-treated L-cells. Somat. Cell Mol. Genet. 1996, 22, 81-85. [CrossRef]

16. Wang, J.K.; Xiang, H.; Liu, L.Q.; Kong, M.H.; Yin, T.; Zhao, X.B. Mitochondrial haplotypes influence metabolic traits across bovine interand intra-species cybrids. Sci. Rep. 2017, 7, 4179. [CrossRef]

17. Wang, M.S.; Li, Y.; Peng, M.S.; Zhong, L.; Wang, Z.J.; Li, Q.Y.; Tu, X.L.; Dong, Y.; Zhu, C.L.; Wang, L.; et al. Genomic Analyses Reveal Potential Independent Adaptation to High Altitude in Tibetan Chickens. Mol. Biol. Evol. 2015, 32, 1880-1889. [CrossRef]

18. Hudson, N.J.; Bottje, W.G.; Hawken, R.J.; Kong, B.; Okimoto, R.; Reverter, A. Mitochondrial metabolism: A driver of energy utilisation and product quality? Anim. Prod. Sci. 2017, 57, 2204-2215. [CrossRef]

19. Himly, M.; Foster, D.N.; Bottoli, I.; Iacovoni, J.S.; Vogt, P.K. The DF-1 chicken fibroblast cell line: Transformation induced by diverse oncogenes and cell death resulting from infection by avian leukosis viruses. Virology 1998, 248, 295-304. [CrossRef]

20. Bacman, S.R.; Moraes, C.T. Transmitochondrial technology in animal cells. Methods Cell Biol. 2007, 80, 503-524.

21. Trounce, I.A.; Kim, Y.L.; Jun, A.S.; Wallace, D.C. Assessment of mitochondrial oxidative phosphorylation in patient muscle biopsies, lymphoblasts, and transmitochondrial cell lines. Methods Enzymol. 1996, 264, 484-509. [PubMed]

22. Grantham, R. Amino-Acid Difference Formula to Help Explain Protein Evolution. Science 1974, 185, 862-864. [CrossRef] [PubMed]

23. Li, W.H.; Wu, C.I.; Luo, C.C. Nonrandomness of Point Mutation as Reflected in Nucleotide Substitutions in Pseudogenes and Its Evolutionary Implications. J. Mol. Evol. 1984, 21, 58-71. [CrossRef] [PubMed]

24. Tamura, K.; Stecher, G.; Peterson, D.; Filipski, A.; Kumar, S. MEGA6: Molecular Evolutionary Genetics Analysis version 6.0. Mol. Biol. Evol. 2013, 30, 2725-2729. [CrossRef] [PubMed]

25. Guo, W.; Jiang, L.; Bhasin, S.; Khan, S.M.; Swerdlow, R.H. DNA extraction procedures meaningfully influence qPCR-based mtDNA copy number determination. Mitochondrion 2009, 9, 261-265. [CrossRef]

26. Livak, K.J.; Schmittgen, T.D. Analysis of relative gene expression data using real-time quantitative PCR and the 2 (T) (-Delta Delta C) method. Methods 2001, 25, 402-408. [CrossRef]

27. Fernandez-Moreno, M.; Soto-Hermida, A.; Vazquez-Mosquera, M.E.; Cortes-Pereira, E.; Relano, S.; Hermida-Gomez, T.; Pertega, S.; Oreiro-Villar, N.; Fernandez-Lopez, C.; Garesse, R.; et al. Mitochondrial DNA haplogroups influence the risk of incident knee osteoarthritis in OAI and CHECK cohorts. A meta-analysis and functional study. Ann. Rheum. Dis. 2017, 76, 1114-1122. [CrossRef]

28. Kenney, M.C.; Chwa, M.; Atilano, S.R.; Falatoonzadeh, P.; Ramirez, C.; Malik, D.; Tarek, M.; Caceres-del-Carpio, J.; Nesburn, A.B.; Boyer, D.S.; et al. Inherited mitochondrial DNA variants can affect complement, inflammation and apoptosis pathways: Insights into mitochondrial-nuclear interactions. Hum. Mol. Genet. 2014, 23, 3537-3551. [CrossRef]

29. Song, M.; Han, W.; Bao, H.; Liu, C.; Wu, C.; Zhao, C. Physiological Adaptability of Tibet Chicken Embryo Liver to Hypoxia and its Protein Profile Analysis. Asian J. Anim. Vet. Adv. 2010, 5, 547-556. [CrossRef]

30. Schon, E.A. Mitochondrial genetics and disease. Trends Biochem. Sci. 2000, 25, 555-560. [CrossRef] 
31. Burton, R.S.; Pereira, R.J.; Barreto, F.S. Cytonuclear Genomic Interactions and Hybrid Breakdown. Annu. Rev. Ecol. Evol. Syst. 2013, 44, 281-302. [CrossRef]

32. Chou, J.Y.; Leu, J.Y. The Red Queen in mitochondria: Cyto-nuclear co-evolution, hybrid breakdown and human disease. Front. Genet. 2015, 6, 187. [CrossRef] [PubMed]

33. Gan, L.; Yan, J.; Liu, Z.J.; Feng, M.; Sun, C. Adiponectin Prevents Reduction of Lipid-Induced Mitochondrial Biogenesis via AMPK/ACC2 Pathway in Chicken Adipocyte. J. Cell Biochem. 2015, 116, 1090-1100. [CrossRef]

34. Reznick, R.M.; Shulman, G.I. The role of AMP-activated protein kinase in mitochondrial biogenesis. J. Physiol. 2006, 574, 33-39. [CrossRef] [PubMed]

35. Cogliati, S.; Frezza, C.; Soriano, M.E.; Varanita, T.; Quintana-Cabrera, R.; Corrado, M.; Cipolat, S.; Costa, V.; Casarin, A.; Gomes, L.C.; et al. Mitochondrial cristae shape determines respiratory chain supercomplexes assembly and respiratory efficiency. Cell 2013, 155, 160-171. [CrossRef] [PubMed]

36. Mishra, P.; Carelli, V.; Manfredi, G.; Chan, D.C. Proteolytic cleavage of Opa1 stimulates mitochondrial inner membrane fusion and couples fusion to oxidative phosphorylation. Cell Metab. 2014, 19, 630-641. [CrossRef]

37. Bellizzi, D.; D’Aquila, P.; Giordano, M.; Montesanto, A.; Passarino, G. Global DNA methylation levels are modulated by mitochondrial DNA variants. Epigenomics 2012, 4, 17-27. [CrossRef]

38. Havird, J.C.; Sloan, D.B. The Roles of Mutation, Selection, and Expression in Determining Relative Rates of Evolution in Mitochondrial versus Nuclear Genomes. Mol. Biol. Evol. 2016, 33, 3042-3053. [CrossRef]

(C) 2020 by the authors. Licensee MDPI, Basel, Switzerland. This article is an open access article distributed under the terms and conditions of the Creative Commons Attribution (CC BY) license (http://creativecommons.org/licenses/by/4.0/). 